$\xi=-1$

\title{
Indentation of rigid rough surface into polymer coating layer
}

\author{
${\text { Petr } \text { Ogar }^{1 *} \text {, Artem Kozhevnikov }}^{1}$, Vladimir Elsukov ${ }^{1}$ \\ ${ }^{1}$ Bratsk State University, 40, Makarenko St., Bratsk, 665709, Russia \\ *Corresponding author E-mail:ogar@brstu.ru
}

\begin{abstract}
An analysis of engineering approaches to determining the value of the introduction of spherical asperity and a rough surface into the polymer layer is given. It is shown that engineering methods of solving contact problems on the basis of simplifying hypotheses are more practical, for example, the representation of a layered body as a construction with special mechanical properties that depend on the mechanical properties of base and coating materials and the thickness of the coating. Analysis of the use of the proposed engineering solutions in calculating the value of the indentation of the sphere showed the advantages of the method based on the rigid model of a layered body. In this case, the effective modulus of elasticity and the Poisson's ratio are determined for any values of the coating thickness for an axisymmetric loading of a layered half-space. To determine the value of the indentation of a rough surface into the polymer layer, a discrete model of a rough surface is used. When contacting a rough surface through a polymer layer, it was taken into account that each asperity corresponds to a certain modulus of elasticity, which is determined by the level of the peak and the value of approach.
\end{abstract}

Keywords: polymeric coating, layered half-space, variable modulus of elasticity, spherical asperity, indentation of sphere, rough surface.

\section{Introduction}

In the sealing technology, metal-polymer seals are widely used if the temperature range permits [1-3]. In this case, it becomes necessary to solve a number of complex problems associated with ensuring minimum pressure on the sealant while maintaining a predetermined tightness of the joint, a minimum coefficient of friction of couplings operating over a wide temperature range, under conditions of increased media pressure and vacuum. One of the promising directions that increase the efficiency of seals and friction units is the application of polymer coatings on their working surfaces or the use of thin polymer films [3]. Experience in the operation of seals and friction units with such coatings shows that their performance is determined not only by the properties of the coating material, but also by its thickness.

The presence of a coating involves taking into account the change in mechanical properties as a function of the distance to the surface. Within the framework of the theory of elasticity, this means that we must consider an elastic body with varying values of the elastic modulus and Poisson's ratio [4]. Contact problems for bodies with mechanical properties that vary in depth have been examined by many researchers [3-7, etc.]. According to the author [4], research methods can be divided into three groups: analytical, numerical and numerically-analytical. However, it is not possible to apply the results obtained to solve practical problems of friction, wear and tightness. An exception is the paper [3], in which the solution of the spatial axisymmetric problem is given in a form accessible for practical use. In this regard, we should also to note [8], in which an approximate solution of the axisymmetric contact problem is given for an elastic layer of finite thickness.

Engineering methods for solving contact problems on the basis of simplifying hypotheses, for example, the representation of a layered body as a topocomposite-constructions with special mechani- cal properties, depending on the mechanical properties of the base and coating materials, the thickness of the coating, should be included in a separate group. In [9], it was proposed to use the Hertz theory for this purpose. On the basis of reliable results for the extreme values of the coating thickness and using the two-point Padé approximation, an expression is obtained for the dimensionless elastic geometric parameter by means of which the elastic constant of the topocomposite and all the main characteristics are determined for its axisymmetric loading.

The authors of [10-12], with the development of the method on the basis of the stiffness model of a layered body, determined the effective modulus of elasticity and the Poisson's ratio for any values of the coating thickness for an axisymmetric loading of a layered semi-space. A classical approach based on the use of potential Boussinescu functions was used to move any point along the symmetry axis into a homogeneous half-space. Using the achievements of previous works [10-12], the author of [13] proposed a simplified model of a layered body and it is an alternative and simpler engineering solution.

The purpose of this paper is to use the solution for a particular spherical asperity to determine the indentation of a rigid rough surface into the polymer layer.

\section{Analysis of engineering approaches to de- termining the indentation of a sphere into the polymer layer}

Consider a sphere of radius $R$ with a layered half-space consisting of a base with elastic modulus $E_{0}$ and a Poisson's ratio $v_{0}$ and a coating with a thickness $\delta$ of elastic modulus $E_{0}$ and a Poisson's 
ratio $v_{1}$. When the sphere is indentated by value $w$, a contact area of radius $a$ is formed. The contact diagram is shown in Fig. 1 .

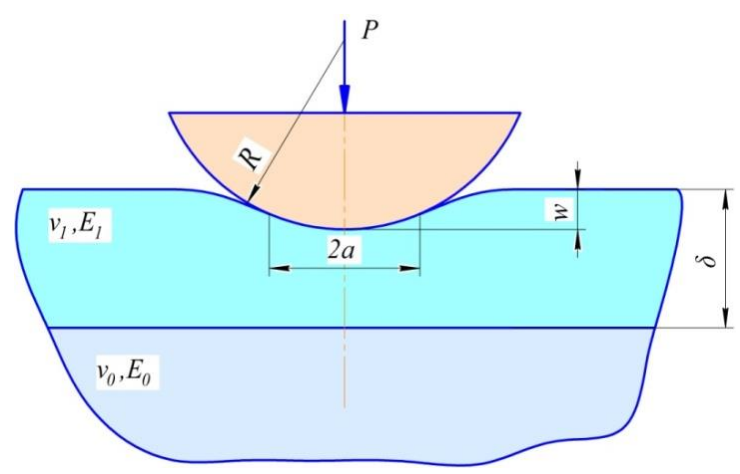

Fig. 1: Scheme of contact of a sphere with a layered half-space

According to the approximate solution of the problem of the introduction of a sphere of radius $\mathrm{R}$ into an elastic layer of thickness $\delta$ lying on a rigid base [8], we have

$P=\frac{8 \theta a^{3}}{3 R}+\frac{16 \theta \delta^{2} a}{\pi} \cdot \frac{N_{3}(a / \delta)}{1-N_{1}(a / \delta)}$

$w=\frac{a^{2}}{R}-\frac{4 \delta a}{R} \cdot \frac{N_{2}(a / \delta)}{1-N_{1}(a / \delta)}$,

where $P$ is the force applied to the sphere; $w$ is the amount of penetration; $a$ is the radius of the contact area; $\theta=E_{1} /\left[2\left(1-v_{1}^{2}\right)\right]$,

$E$ and $v$ are the modulus of elasticity and the Poisson's ratio of the elastic layer.

We introduce the notation: $\alpha=a / \delta, \bar{\delta}=\delta / R, \bar{w}=w / \delta$, $E^{*}=E /\left(1-v^{2}\right), \bar{P}=P /\left(E^{*} R^{2}\right)$ and represent the expressions (1) and (2) in the form:

$\bar{P}(\alpha, \bar{\delta})=\frac{4}{3} \alpha^{3} \bar{\delta}^{3}+\frac{8}{\pi} \alpha \bar{\delta}^{3} \cdot \frac{N_{3}(\alpha)}{1-N_{1}(\alpha)}$,

$\bar{w}(\alpha, \bar{\delta})=\alpha^{2} \bar{\delta}-\frac{4}{\pi} \alpha \bar{\delta} \cdot \frac{N_{2}(\alpha)}{1-N_{1}(\alpha)}$,

$N_{i}(\alpha)$ is functions given in [8], are defined by the expressions:

$$
\begin{aligned}
& N_{1}(\alpha)=\frac{2}{\pi} \cdot \int_{0}^{\infty}[1-L(u)] \cdot \operatorname{Si}(\alpha u) \cdot\left(\frac{\sin \alpha u}{\alpha u}-\cos \alpha u\right) \cdot \frac{d u}{u} ; \\
& N_{2}(\alpha)=\int_{0}^{\infty}[1-L(u)] \cdot\left(\frac{\sin \alpha u}{\alpha u}-\cos \alpha u\right) \cdot \frac{d u}{u^{2}} ; \\
& N_{3}(\alpha)=\int_{0}^{\infty}[1-L(u)] \cdot\left(\frac{\sin \alpha u}{\alpha u}-\cos \alpha u\right) \cdot \sin \alpha u \cdot \frac{d u}{u^{3}} ; \\
& L(u)=\frac{2 k \cdot \operatorname{sh}(2 u)-4 u}{2 k \cdot \operatorname{sh}(2 u)+1+k^{2}+4 u^{2}} ; k=3-4 v ; \\
& \operatorname{Si}(x)=\int_{0}^{x} \frac{\sin t}{t} d t .
\end{aligned}
$$

We define analogous dependences for the indentation of a sphere into the stratified half-space, using the engineering solution from $[11,13]$.
The effective modulus of elasticity is determined by equations

$E_{01}^{*}=E_{1}^{*} \cdot F$

$F=F\left(\bar{z}, I_{e}\right)=\frac{\pi / 2}{\pi / 2-K_{1}(\bar{z})+K_{0}(\bar{z}) \cdot I_{e}}$

$K_{i}(\bar{z})=\operatorname{arcctg} \bar{z}+\frac{v_{i}}{1-v_{i}} \bar{z}(1-\bar{z} \cdot \operatorname{arcctg} \bar{z}) ;$

where $I_{e}=\frac{E_{1}^{*}}{E_{0}^{*}}=I \cdot \frac{1-v_{0}^{2}}{1-v_{1}^{2}}, I=\frac{E_{1}}{E_{0}} ; \bar{z}=\frac{z}{a}$.

For the case of contact between a rigid sphere and a layered halfspace, the approach and radius of the contact spot are determined by equations

$w_{01}=w_{1} \cdot F_{1}^{-\frac{2}{3}}, \quad a_{01}=a_{1} F^{-\frac{1}{3}}$.

From the Hertz formula for the radius of the contact spot, we have

$\bar{P}=\frac{P}{E^{*} R^{2}}=\frac{4}{3} \frac{a^{3}}{R^{3}}$

With taking into account the above notations and Eqs. (5), (6), (8) and (9), we have

$$
\bar{P}(\alpha, \bar{\delta})=\frac{4}{3} \alpha^{3} \bar{\delta}^{3} F\left(\alpha, I_{e}\right), \quad \bar{w}(\alpha, \bar{\delta})=\alpha^{2} \bar{\delta} \cdot\left[F\left(\alpha, I_{e}\right)\right]^{-\frac{2}{3}}
$$

where $\alpha=\bar{z}^{-1}=a / \delta$.

When $F=1$ Eq. (10) describes the contact of the sphere with a half-space of the coating material.

Let's consider other engineering approaches. Using the adopted notation, the Eq. (2.39) from [3, p. 69] can be represented as

$F_{1}\left(\alpha, I_{e}\right)=\left(\frac{I_{e}^{1.33}+0.8 \bar{z}(\alpha)}{\sqrt{1+(0.8 \bar{z}(\alpha))^{2}}}\right)^{\frac{-3}{4}}, \quad \bar{z}(\alpha)=\alpha^{-1}$

Similarly, from the expression (20) of [9], we have

$$
\begin{aligned}
& F_{1}\left(\alpha, I_{e}\right)= \\
& I_{e}^{-1} \cdot\left[\begin{array}{l}
I_{e}^{\frac{-2}{3}}+\left(1-I_{e}^{\frac{-2}{3}}\right) \times \\
\left.\times\left(\frac{1+1.27 \bar{z}(\alpha)^{3} I_{e}^{-1}-0.333 \bar{z}(\alpha)^{2} I_{e}^{\frac{-2}{3}}-0.424 \bar{z}(\alpha) I_{e}^{\frac{-1}{3}}}{1+2 \bar{z}(\alpha)^{4} I_{e}^{\frac{-4}{3}}}\right)\right]
\end{array}\right]
\end{aligned}
$$

Eqs. (11) and (12) are identical in their physical essence to Eq. (6) and determine the change in the effective modulus of elasticity, depending on the elastic properties of the coating and base materials, and also on the thickness of the coating.

In Fig. 2 shows the dependencies of relative penetration on the relative load: curve 1 corresponds to the Eq. (10); curve 2 to the Eqs. (3) and (4); curves 3 and 4 - according to the Eqs. (11) and (12); curve 5 corresponds to the introduction of a sphere into an elastic half-space of the coating material. 


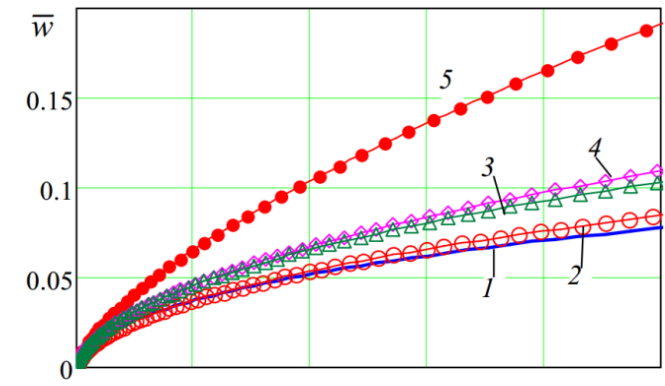

a) $0 \quad 2 \times 10^{-3} \quad 4 \times 10^{-3} \quad 6 \times 10^{-3} \quad 8 \times 10^{-3} \quad \bar{P}$

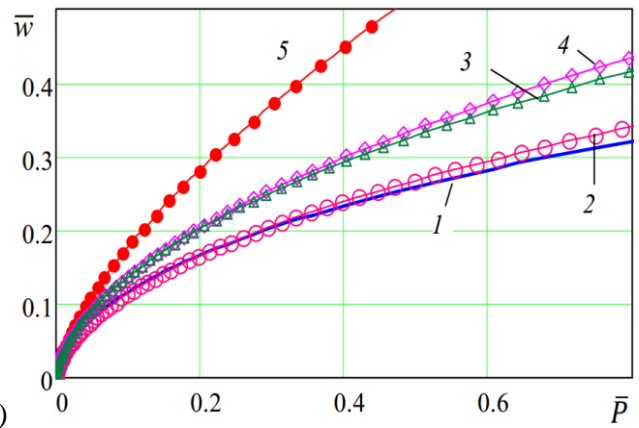

Fig. 2: Dependences of relative introduction of the relative load:

$$
\text { (a) } \bar{\delta}=0.2 \text {; (b) } \bar{\delta}=1 \text {. }
$$

\section{Contact rough surfaces through a layer of polymer coating}

Let us use the discrete model of a rough surface, in which microasperities are presented by identical spherical segments with the distribution of segments' peaks on height corresponds to the bearing profile curve of the real surface $[14,15]$. To describe the distribution of the bearing curve is used incomplete beta function

$$
\eta(\varepsilon)=\frac{\mathrm{B}_{\varepsilon}(p, q)}{\mathrm{B}(p, q)}
$$

where $\mathrm{B}_{\varepsilon}(p, q)$ and $\mathrm{B}(p, q)$ are incomplete and complete beta functions;

$$
p=\left(\frac{R_{p}}{R_{q}}\right)^{2}\left(\frac{R_{\max }-R_{p}}{R_{\max }}\right)-\frac{R_{p}}{R_{\max }}, q=p\left(\frac{R_{\max }}{R_{p}}-1\right) .
$$

where $R_{p}, R_{q}, R_{\max }$ are height roughness parameters according to ISO $4281 / 1-1997$.

In this case, the density of the asperities distribution on height function is

$$
\varphi_{n}^{\prime}(u)=\frac{u^{p-2}(1-u)^{q-2}[(p-1)(1-u)-(q-1) u]}{\varepsilon_{s}^{p-1}\left(1-\varepsilon_{s}\right)^{q-1}},
$$

where $\varepsilon_{s}$ is determined from the condition $\varphi_{n}\left(\varepsilon_{s}\right)=1$ [14].

Geometrical parameters of a spherical segment are: the height is $\omega R_{\max }$, where $\omega=1-\varepsilon_{s} ; a_{c}$ is the radius of the base of the spherical segment.

$$
R=\frac{a_{c}^{2}}{2 \omega R_{\max }}
$$

where $R>R_{\max }$.
When describing the bearing curve of the profile to the level of the midline with $\varepsilon_{p}=R_{p} / R_{\max }$ a parabola

$$
\begin{aligned}
& \eta(\varepsilon)=t_{p}(\varepsilon)=b \varepsilon^{v}, \\
& b=t_{m}\left(R_{\max } / R_{p}\right)^{v}, \quad v=2 t_{m} \frac{R_{p}}{R_{a}}-1 .
\end{aligned}
$$

where $t_{m}=t_{p}\left(\varepsilon_{p}\right)$.

In this case, the function and density of the height distribution function of the asperities are described by expressions

$$
\varphi_{n}(u)=\left(\frac{u}{\varepsilon_{s}}\right)^{v-1} ; \quad \varphi_{n}^{\prime}(u)=\frac{(v-1) u^{v-2}}{\varepsilon_{s}^{v-1}}
$$

To analyze the results obtained using different reference curves, it is important to know the relationships between the parameters $p$ and $q$ when describing the bearing curve of the profile with the incomplete beta function, and the parameters $v$ and $b$ in the description of the bearing curve by the parabola, which are determined by the following the following expressions [14]:

$$
v=\frac{\mathrm{B}_{\varepsilon_{p}}(\alpha+1, \beta)}{\varepsilon_{p} \mathrm{~B}_{\varepsilon_{p}}(\alpha, \beta)-\mathrm{B}_{\varepsilon_{p}}(\alpha+1, \beta)}, b=\frac{\mathrm{B}_{\varepsilon_{p}}(\alpha, \beta)}{\mathrm{B}_{\varepsilon_{p}}(\alpha+1, \beta)}\left(\frac{\alpha+\beta}{\alpha}\right)^{v}
$$

here $\varepsilon_{p}=\frac{R_{p}}{R_{\max }}=\frac{\alpha}{\alpha+\beta}$.

The scheme of the interaction of a single asperity is shown in Fig. 1. Assuming that the distribution function of the asperities $\varphi_{n}(u)$ continuous, the number of asperities in the layer between the levels $u$ and $u+d u$

$d n_{r}=n_{c} \varphi_{n}^{\prime}(u) d u$

where $n_{c}=A_{c} /\left(\pi a_{c}^{2}\right)$, is the contour area.

Using the solution of the Hertz problem on the introduction of a sphere by an amount $h_{i}=R_{\max }(\varepsilon-u)$ in the elastic half-space, determine the necessary force for this $N_{i}$

$N_{i}=\frac{4}{3} E_{01}^{*} R^{\frac{1}{2}} R_{\max }^{\frac{2}{3}}(\varepsilon-u)^{\frac{3}{2}}$

Summing over all the asperities, we obtain

$N=\frac{8}{3} n_{c} a_{c} \omega R_{\max } \int_{0}^{\min \left(\varepsilon, \varepsilon_{s}\right)} E_{01 i}^{*}\left(\frac{\varepsilon-u}{2 \omega}\right)^{\frac{3}{2}} \varphi_{n}^{\prime}(u) d u$

Effective modulus of elasticity when contacting a particular asperity $E_{01 i}^{*}$ is defined similarly to Eqs. (5) - (7).

The amount $\bar{z}_{i}$ in the form:

$\bar{z}_{i}=\frac{\delta}{a_{i}}=\frac{\delta}{a_{c}} \cdot \frac{a_{c}}{a_{r i}}=\gamma \cdot \eta_{i}^{-0.5}$

where $\gamma=\delta / a_{c}, a_{i} \equiv a_{r i}, \eta_{i}=a_{r i}^{2} / a_{c}^{2}=\frac{\varepsilon-u}{2 \omega}$.

Then 


$$
\begin{aligned}
& F_{i}=F_{i}\left(\gamma, \eta_{i}, I_{e}\right)=F_{i}\left(\gamma, \varepsilon, u, I_{e}\right)= \\
& =\frac{\pi / 2}{\pi / 2-K_{1}(\gamma, \varepsilon, u)+K_{0}(\gamma, \varepsilon, u) \cdot I_{e}}
\end{aligned}
$$

Values $K_{1}(\gamma, \varepsilon, u)$ are calculated from Eq. (7) with allowance for (23). Substituting Eq. (24) into (22) and taking into account that $q_{c}=N / A_{c}$, we get

$$
\begin{aligned}
& q_{c}=q_{c}\left(\gamma, \varepsilon, I_{e}\right)=\frac{8}{3 \pi} \cdot \frac{\omega R_{\max }}{a_{c}} E_{1}^{*} \times \\
& \times \int_{0}^{\min \left(\varepsilon, \varepsilon_{s}\right)} F_{i}\left(\gamma, \varepsilon, u, I_{e}\right) \cdot\left(\frac{\varepsilon-u}{2 \omega}\right)^{\frac{3}{2}} \varphi_{n}^{\prime}(u) d u,
\end{aligned}
$$

or in dimensionless form

$$
\begin{aligned}
& F_{q 1}=F_{q 1}\left(\gamma, \varepsilon, I_{e}\right)=\frac{q_{c} a_{c}}{\omega R_{\max } E_{1}^{*}}= \\
& =\frac{8}{3 \pi} \cdot \int_{0}^{\min \left(\varepsilon, \varepsilon_{s}\right)} F_{i}\left(\gamma, \varepsilon, u, I_{e}\right) \cdot\left(\frac{\varepsilon-u}{2 \omega}\right)^{\frac{3}{2}} \varphi_{n}^{\prime}(u) d u .
\end{aligned}
$$

In Fig. 2 shows the relationship between the rough surface and the polymer layer as a function of the nominal pressure, calculated in the Mathcad in Eq. (25). The dots denote previously "digitized" experimental data given in [3, Fig. 3.14]. The following roughness parameters were used in the calculations: $R_{\max }=8.25 \mu \mathrm{m}$, $R=20 \mu m, b=1.9, v=1.59$.

a)
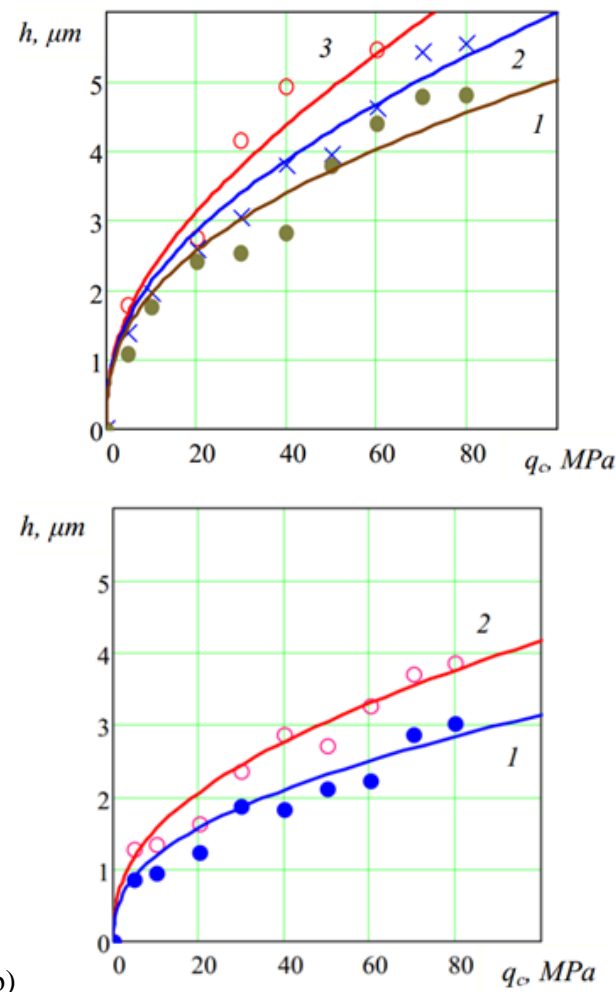

Fig. 3: Dependences of rough surface and polymer layer approach from the nominal pressure: a - fluoroplastic coating ФБФ-74Д ( 1 - at $\delta=12 \mu \mathrm{m}$; 2 - at $\delta=25 \mu \mathrm{m} ; 3$ - bulk material); b - lavsan films $\delta=25 \mu \mathrm{m}$ (curve 1$)$ и polyamide $\mathrm{PM}-1, \delta=45 \mu \mathrm{m}(2)$.

\section{The conclusion}

1. Analysis of the proposed engineering solutions for the indentation of the sphere showed the advantages of the method based on the rigid model of a layered body. In this case, the effective modulus of elasticity and the Poisson's ratio are determined for any values of the coating thickness for an axisymmetric loading of a layered half-space.

2. A layered body with a polymeric coating is presented as a structure with special mechanical properties depending on the mechanical properties of the base and coating materials and the thickness of the coating.

3. When investigating the contact of a rough surface through a polymer layer, it should be taken into account that each asperity corresponds to a certain modulus of elasticity, which is determined by the level of the peak and the magnitude of the approach.

4. The proposed engineering solution for changing the effective modulus of elasticity is well combined with a discrete roughness model which allows to successfully determine any characteristics in the interaction of a rough surface with a layered body, for example, the relative contact area [16] or the gap density in the joint [17].

5. The obtained results shows high sensitivity of the proposed method to a change in the thickness of the coating and its mechanical properties. The results of calculations and experimental data on the indentation of a rough surface into polymer coatings are in satisfactory agreement.

\section{References}

[1] Golubev AI \& Kondakov LA editors, Handbook of seals and sealing equipment. 2nd ed. Mechanical Engineering, Moscow, (1994), $448 \mathrm{p}$.

[2] Ogar PM, Sheremeta RN \& Lkhanag D (2006), The tightness of the metal-polymeric joints of rough surfaces, BrSU, Bratsk, $159 \mathrm{p}$.

[3] Makushkin AP, Polymers in friction and seal applications at low temperatures, Mechanical Engineering, Moscow, (1993), 288 p.

[4] Torskaya EV, Modeling of frictional interaction of bodies with coverings. Dissertation, Moscow, (2014), 251 p.

[5] Aleksandrov VM \& Mkhitaryan SM (1983), Contact problems for bodies with elastic coatings and interlayers, Nauka, Moscow, 488p.

[6] Aizikovich SM, Aleksandrov VM, Vasiliev AS, Krenev LI \& Trubchik IS (2011), Analytic solutions of mixed axisymmetric problems for functional gradient media, Fizmatlit, Moscow, 192 p.

[7] Potelezhko VP (2006), The Bousinescu problem for a two-layer half-space, Mechanics and physics of processes on the surface and in the contact of solid bodies and machine parts 2, 27-32.

[8] Argatov II (2004), Approximate solution of an axisymmetric contact problem for an elastic layer of finite thickness, Problems of mechanical engineering and reliability of machines 6, 35-40.

[9] Voronin NA (1993), Application of the theory of the elastic Hertz contact to the calculation of the stress-strain state of a layered elastic body, Friction and Wear 14, 250-258.

[10] Ogar PM, Tarasov VA \& Fedorov IB (2013), Control of stiffness of contact systems of sealing joints, Modern technologies. System analysis. Modelin 1, 22-27.

[11] Ogar PM \& Tarasov VA (2013), Determination of the Elastic Characteristics of Bodies with Thin Coatings, Advanced Materials Research 677, 267-272. doi: 10.4028/www.scientific.net/AMR 677.267.

[12] Ogar PM, Gorokhov DB \& Kozhevnikov AS (2016), Effective modulus of elasticity of a layered body. Modern technologies. System analysis. Modeling 4(52), 37-42.

[13] Kozhevnikov AS (2016), A new engineering solution for determining the effective modulus of elasticity of a layered body, Proceedings of the Bratsk State University. Series: Natural and engineering sciences 2, 70-76.

[14] Ogar PM, Gorokhov DB \& Kozhevnikov AS (2017), Contact problems in hermetic sealing studies of fixed joints, BrSU, Bratsk, $242 \mathrm{p}$,

[15] Ogar P, Gorokhov D \& Ugryumova E, (2017) Mechanics of unloading of a rough surfaces pre-loaded joint, MATEC Web of Conferences 129, 06016. doi.org/10.1051/matecconf/201712906016

[16] Ogar PM, Tarasov VA \& Gorokhov DB (2015), The influence of coating thickness on the relative area of tribounits contact, $\mathrm{Ad}$ vanced Materials Research 1061-1062, 614-618. doi: 10.4028/www.scientific.net/AMR.1061-1062.614

[17] Ogar PM, Gorokhov DB \& Kozhevnikov AS (2015), The density of gaps in the seal joint in elastic contact of microasperities, Proc. 2nd International Conference on Modelling, Identification and Control (MIC 2015), 177-180. doi:10.2991/mic-15.2015.40. 